\title{
Die Prägung Japans durch das Klima
}

\section{Einleitung}

"Guten Tag" heisst auf Japanisch "kon nichi wa". Dieser Gruss wird im Sommer häufig ersetzt durch "atsui desu, nê?" (= heiss ist es, nicht wahr?) und im Winter durch "samui desu, nê?" (= kalt ist es, nicht wahr?). Japan hat erschöpfend heisse Sommer und beissend kalte bis empfindlich kühle Winter. Die Temperaturschwankung (hier: die Differenz der Monatsmittelwerte von August und Januar) ist um $10-150 \mathrm{C}$ grösser als in Mitteleuropa (Abb.1). "Bambus im Schnee" (M. SCHWIND 1967) ist eine aussagekräftige Assoziation zum Klima von Japan.

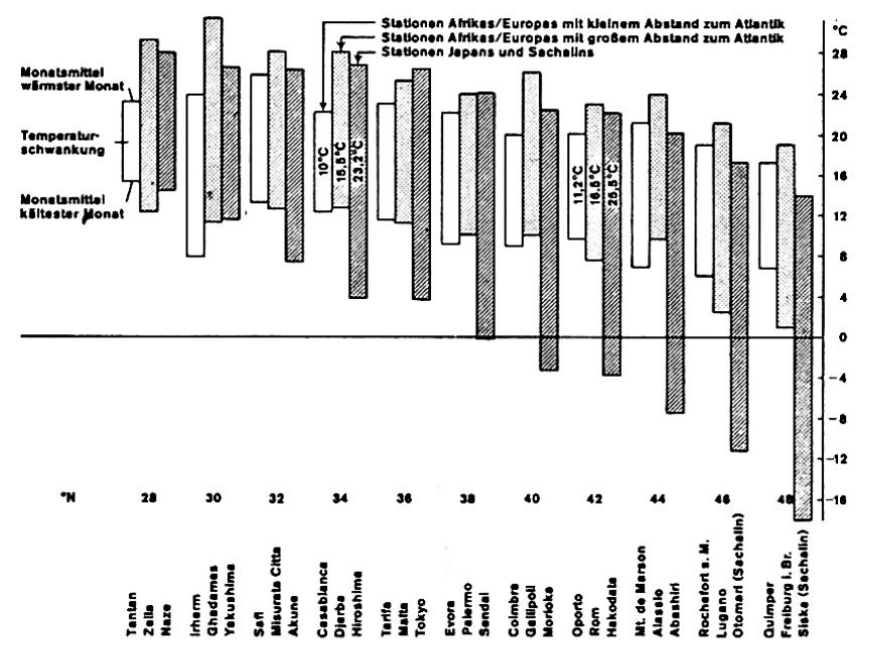

Abb.1: Temperaturschwankungen. Vergleich von Stationen Japans mit Stationen Afrikas/Europas auf gleicher Geogr. Breite

Im Frühling und Sommer werden den japanischen Inseln tropische Luftmassen von Süden und Südwesten zugeführt. Die Mitteltemperaturen steigen auf $26-28^{\circ} \mathrm{C}$ in Kyushu und erreichen in Hokkaido 18 - $22^{\circ} \mathrm{C}$. Ein grosser Teil des Landes ist im Sommer so heiss wie äquatornahe Gebiete und der Norden immerhin noch wärmer als Mitte1europa zur selben Zeit.

Im Herbst verändert sich das Windregime; die Winde wehen aus dem sich zusehends abkühlenden ostsibirischen Raum. Woh1 wird die Kälte der Luftmassen beim Ueberstreichen der $300-700 \mathrm{~km}$ breiten Japan Sea etwas gemildert, doch sinken die winterlichen Temperaturen in Japan, dessen Hauptinseln zwischen $32^{\circ}$ und $44^{\circ} \mathrm{N}$ liegen, we- sentlich tiefer, als es in Hinblick auf die Breitenlage zu erwarten wäre. Die Tieflands-Mitteltemperaturen betragen im Januar in Hokkaido -5 - $-10^{\circ} \mathrm{C}$, in Zentral-Honshu $0-4{ }^{\circ} \mathrm{C}$ und bleiben nur im äussersten Süden über $10^{\circ} \mathrm{C}$.

Es ist also ein ausgeprägter Sommer-Winter-Gegensatz der Temperaturen festzustellen und eine starke Abnahme der jährlich zugeführten Wärmemengen von Süden nach Norden. Die südlichsten Inseln Japans sind von Korallenriffen gesäumt; im Nordosten Hokkaidos erreicht Treibeis beinahe alljährlich die Küste.

Japan, der gebirgige Archipel, ist ganzjährig humid. Der Gang der Niederschläge ist allerdings nicht ausgeglichen. Wenn im Frühling die kalten Luftmassen nach Norden zurückgeschoben werden und die Polarfront über das Land nach Nordosten wandert, treten im Grenzbereich der tropischpazifischen und der subpolar-sibirischen Luft verstärkte Niederschläge auf. Es ist die Regenzeit bai-u. Umgekehrt drängt im Herbst die nordische Kaltluft die tropisch-pazifische nach Süden und die Regenzeit shurin quert das Land, im südlichen Teil überlagert und gefolgt von Taifunen und damit heftigen Regengüssen. Die Sommerwinde sind für das ganze Land Niederschlagsspender; die Winterwinde hingegen nicht. In Honshu laden sie ihre über der Japan Sea aufgenommene Feuchtigkeit in Form reicher Schneefälle beim Auftreffen aufs Land und dem Steigen an den Gebirgshängen ab. Die pazifische Seite bleibt während dieser Zeit weitgehend trocken und wolkenfrei.

Un in der Folge die Prägung Japans durch das Klima regional differenziert betrachten zu können, empfiehlt sich eine einfache Gliederung Japans in vier Klimaprovinzen (Abb.2):

Südprovinz:

- Sommer: heiss, niederschlagsreich, schwül

- Winter: mild, im Tiefland dauernd frostfrei, mässige Niederschläge

relativ kleine Gegensätze zwischen den einzelnen Jahreszeiten; subtropischer frost- und schneefreier Teil Japans

Japan Sea Provinz Honshus:

- Sommer: heiss bis warm, niederschlagsreich, schwül

- Winter: mild bis kühl, hohe Schnee-Niederschläge

schneereicher Teil Japans

Dr. Ludwig Ellenberg, Institut für Geographie, TU Berlin, Kurfürstendamm 195, 1000 Berlin 15 
Pazifik Provinz Honshus:

- Sommer: wie Japan Sea Provinz Honshus heiss bis warm, niederschlagsreich, schwül

- Winter: zwar mild bis kühl, aber im Gegensatz zur anderen Seite Honshus sonnig und schneearm

Nordprovinz (Hokkaido):

- Sommer: warm und feucht

- Winter: kalt und lang; im Westen und Zentralteil schneereich, kurze Vegetationszeit

Für alle vier Klimaprovinzen, besonders aber für die beiden auf Honshu gelegenen gilt, dass vier Jahreszeiten tiefgreifend unterschiedlicher Prägung entstehen. So verwundert es nicht, dass die Jahreszeiten in japanischer Prosa und Lyrik mehr als eine Kulisse darstellen. Ganz offenkundig wird das Bewusstsein für die Stimmung der Jahreszeiten in den Haikus, den 17-silbigen Kurzgedichten.

Bitte, mach doch Platz,
dass ich Bambus pflanzen kan
alter Kröterich
"S'ist die Schwüle nur",
sagte sie und fing dabei
still zu weinen an.
Totgetreten liegt
einer Krabbe Leichnam da -
Morgendlicher Herbst
Mit dem letzten Zahn
tau ich meinen Pinsel auf -
Kalte Winternacht
Chora 1726-1781

Kigin 1623-1705

Shiki 1866-1902

Buson 1715-1783

\section{Prägung des Reliefs}

Die utopisch anmutende Topographie auf japanischen Holzschnitten mag zwar nicht vollkommen wirklichkeitsgetreu sein, stellt aber den wesentlichsten Eindruck heraus: Japan ist vom Süden bis etwa zum 40. Breitengrad, also innerhalb des alten Siedlungsraums, ein Gebirgsland mit grossen Höhenunterschieden auf engem Raum und nur kleinen Ebenheiten. In Kyushu, Shikoku und dem westlichen und zentralen Honshu fallen im Innern der Inseln steile Hänge, tiefeingeschnittene Kerbtäler, grobblockige Talalluvionen und sehr kurze Uebergänge von Talhang zu Talboden auf.

Drei Viertel des Landes sind stärker geböscht als $15^{\circ}$ (M. SCHWIND 1967). Bei den vielen Stratovulkanen, die das Relief Japans charakterisieren und der seit dem Mesozoikum nie zur Ruhe gekommenen tektonischen Bewegung überrascht diese Tatsache nicht. Es ist allerdings darauf hinzuweisen, dass die Steilheit der Hänge nicht auf Vulkane beschränkt ist und teilweise nicht auf geologische, sondern auf klimatische Ursachen zurückzuführen ist. Ein Grossteil der Niederschläge geht als Starkregen nieder und trifft auf tiefgründig zersetztes Material (heisse, feuchte Sommer!), auf relativ frische vulkanische Lockerprodukte oder gar auf Tephra (ver-

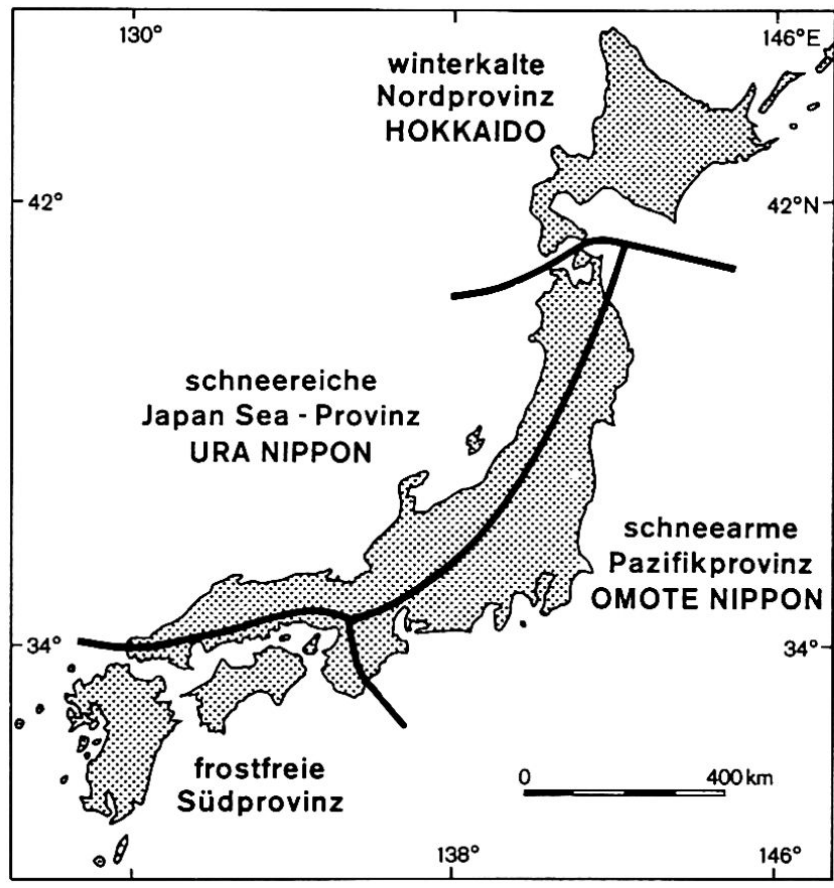

Abb.2: Klimaprovinzen

witterte und dadurch verlehmte Asche). Kurze aber starke Abtragungsleistungen der Wildbäche in den Gebirgen und der Flüsse in den grösseren Talungen sind die Folge. Das Relief ist ein weitgehend fluvial gestaltetes und der activity degree, die Veränderung des Reliefs pro Zeiteinheit, ist generell wesentlich grösser als in Mitteleuropa.

Für Japan als Lebensraum der Menschen ist die starke Erosion im Innern ein Hindernis, - man denke nur an die Anlage von Siedlungen, Reisterrassen und Strassen an den Hängen. Aber an den Rändern der Inseln sind durch Ablagerung der ausgeräumten Massen Deltas und durch die Küstenströmungen vertriftete schmale Tieflandbänder entstanden. Ohne diese Ebenheiten hätte sich Japan nicht zum heutigen Gigant in weltwirtschaftlicher Hinsicht entwickeln können.

Hokkaido und der Norden Honshus (Tohoku) wirken in Bezug auf das Relief unjapanisch weitläufig und grosszügig gestaltet. Flache Hänge und Nuldentäler sind viel häufiger als im Süden. Auch dies ist klimatisch $z u$ interpretieren. Hokkaido ist weitgehend frei von Taifunen und damit morphologisch viel weniger durch plötzliche Abflussereignisse geprägt. Ausserdem wurde es viel stärker als der Süden während der pleistozänen Kaltzeiten durch Gletscher gestaltet und in den eisfreien Gebieten bis zum damaligen Meeresspiegel hinab intensiv durch periglaziale Solifluktion überformt. Hokkaido und Tohoku stellen weniger ein rezent gestaltetes fluviales Relief dar als ein (letzt)kaltzeitliches Glazial- und Periglazialrelief. 


\section{Honshus zwei Seiten}

Die Pazifikseite Honshus ist das dynamischste Gebiet Japans. Hier reihen sich die Städte Tokyo, Kawasaki, Yokohama, Shimizu, Nagoya, Gifu, Kyoto, Osaka, Kobe, Okayama mit den sich verdichtenden Agglomerationen dazwischen aneinander. Hier war der Schauplatz aller grossen kulturellen Leistungen Japans und der Auseinandersetzungen zwischen Tenno und Shogun. Hier begann die Industrialisierung Japans und von hier aus wird Japan heute gesteuert. Diese Seite Honshus heisst Omote Nippon. "Omote" heisst "vorne". Japans Fensterseite blickt auf den Pazifik, nicht auf Festlandasien.

Die Seite Honshus, die zur Japan Sea hin entwässert, heisst Ura Nippon. "Ura" heisst "hinten". Ura Nippon ist das vergessene, vielerorts sehr traditionelle, wirtschaftlich weniger wichtige und langsamere Japan. Honshus Hinterseite liegt dem asiatischen Kontinent gegenüber. Japan hat quasi die Tür hinter sich zugemacht. Dies ist für eine kontinentnahe Insel eine beinahe einmalige Gegebenheit.

Die Asymmetrie Honshus wurde tektonisch-morphographisch erklärt. Omote Nippon hat fast durchgehende Küstenebenen; das Tiefland von Ura Nippon hingegen zerfällt in mehrere Kammern, die nicht ohne weiteres miteinander verbunden werden können. Diese Erklärung ist zwar richtig, nur genügt sie alleine nicht aus. Die unterschiedliche Inwertsetzung der beiden Seiten Honshus wurde in erster Linie klimatisch erzwungen. Die Sommer sind in beiden Teilen ähnlich, die Winter aber grundverschieden. Omote Nippon bleibt im Winter weitgehend schneefrei. Die Kommunikation ist dauernd einfach; der Verkehr wird kaum erschwert. Der Winter in Ura Nippon ist dagegen eine Zeit der totalen Schneeblockade. Er beginnt nicht mit Frost, sondern mit Schneefall. Es schneit mehrere Wochen lang fast ohne Unterbruch. Im Tiefland bauen sich Schneedecken von $2 \mathrm{~m}$ und mehr auf, die drei bis vier Monate überdauern. In den Nordjapanischen Alpen sind Schneedicken von einigen Metern die Regel und bei Verwehungen sind auch $10 \mathrm{~m}$ keine Seltenheit (Mitt. von S. IWATA). Die meisten Häuser haben auffallend steile Dächer, damit der Schnee abrutschen kann; in vielen Bauernhäusern ist der Eingang im Sommer im Parterre, im Winter im 1. Stock.

Der Verkehr wird von November an erschwert und bricht in den Wintermonaten oft zusammen. Ausser für den Wintersport, einem Zweig japanischer Freizeitgestaltung mit hoher Wachstumsrate, hat Ura Nippon für Japan wenig Bedeutung, nicht einmal als Pionierland. Katastrophal kann sich im Frühling und Frühsommer die Schneeschmelze auf die kleinen Ebenen und die flussnahen Siedlungen auswirken. Ura Nippon ist das "yuki no kuni" von Kawabata, das Sclineeland Japans. Es ist eine der schneereichsten Regionen der Erde und dies ist eine Tatsache, der auch eine führende Industrienation nicht voll gewachsen ist.

\section{Vegetation}

Der starke Abfall der Wärmemengen von Südwesten nach Nordosten bedingt eine enge Scharung der Vegetationszonen (M. NUMATA 1974) - viel enger als beispielsweise an der Westseite Eurasiens.

Die Tiefländer Kyushus, Shikokus, West- und Zentral-Honshus gehören in die Zone der Lorbeerwälder, der immergrünen Camelietae japonicae. Von den einstigen Wäldern sind allerdings nur noch äusserst kleine Relikte bei shintoistischen Schreinen und buddhistischen Tempeln übriggeblieben. Es gibt nur wenige Vegetationstypen auf der Erde, die derart stark zurückgedrängt wurden.

Die Wälder der montanen Stufe Zentral-Honshus und der planaren und collinen Tohokus zeigen inmergrüne Arten wegen der längeren und stärkeren Winterkälte nur in ihrem Unterwuchs. Die Bäume bestehen aus vielen sommergrünen Arten (Fagetea crenatae). Nach der Zeit der Taifune, wenn die Winde nicht stark wehen, die Herbstniederschläge abgeklungen sind und die Fröste erst zögernd einsetzen, verfärben sich diese Wälder äusserst bunt. Das nordöstliche Omote Nippon gehört zu den schönsten Herbstlandschaften der Erde. Es ähnelt darin und allgemeinklimatisch dem Nordosten der USA.

Hokkaidos Wälder und die die Waldgrenze bildende Stufe auf den weiter südlich gelegenen Inseln gehören zur Vaccinio-Picetea-japonica-Zone. Gegen Winterkälte resistente Nadelbäume dominieren. Ergänzt werden sie durch Birken und andere Laubbäume, die monatelang unter Schnee begraben sein können. Der Wald ist nur selten hochstämmig. Dichter Bergbambus (sasa sp.) bildet den Unterwuchs. Die Wälder stellen einen starken Kontrast zu den beiden anderen Waldtypen dar.

Weit unterhalb der Waldgrenze schon setzen an Stellen, die nicht dicht bewachsen sind (Rutschungen, Umland von Funarolen, Rodungen), Periglazialformen mit rezenter Formungsaktivität ein, und zwar nicht nur Bülten (earth hummocks), sondern auch Terrassetten (sorted steps) und stellenweise sogar Streifenböden (sorted stripes). Die Untergrenze der periglazialen Stufe ist in Japan also viel schwieriger zu ermitteln als beispielsweise in den europäischen Alpen, den Pyrenäen oder Karpaten. Dieses Phänomen ist mit dem ausgeprägten Jahresgang der Temperatur zu erklären. Die warmen feuchten Sommer lassen den Wald noch in Höhen gedeihen, die während der kalten Jahreszeiten bereits frostwechselinduzierter Formung unterworfen sind (L. ELLENBERG 1974a, 1977). Aehnliches kennt man aus Sibirien und Nordkanada mit einer noch viel grösseren Temperaturschwankung, wo Periglazialformen und wald einander nicht ausschliessen wie in den Gebirgen mit kleiner Temperaturschwankung, sondern im gleichen Areal vorkommen.

Die Waldgrenze sinkt von $2500 \mathrm{~m}$ in Zentral-Honshiu rasch auf ca. $800 \mathrm{~m}$ in Nordost-Hokkaido ab. Meist ișt sie messerscharf ausgebildet; dies 
stellt einen auffallenden Gegensatz zu vielen andèren Gebirgen der gemässigten Breiten dar, wo oft eine Wald- und eine Baungrenze unterschieden werden. Bei diesem Phänomen ist der Grund nicht ein unmittelbar klimatischer. Die Waldauflösung im Bereich der obersten Waldstufe ist oft eine Folge der Almwirtschaft; die Grenze wäre von Natur aus nur ein sehr schmaler Gürtel (H. ELLENBERG 1966). Die anthropogene Beeinflussung drückt die Grenze hinab und lockert die obersten Waldbestände auf. In Japan fehlt traditionell - wie in ganz Ostasien - die Grossviehhaltung. Ueberdies wäre eine Almwirtschaft europäischer Prägung unmöglich, denn es fehlen die dafür notwendigen Trogschultern (schwächere und nicht so weit hinabreichende glaziale Prägung Honshus als der europäischen Alpen!) und die Steilhänge bieten für einen eventuellen Vieh-Auftrieb besondere Probleme. Ausserdem wird die landwirtschaftliche Fläche in den tiefgelegenen Tälern ökonomischer mit hochwertigeren Produkten bestellt als mit Gras für die Winterfütterung von Kühen. Nur Hokkaido eignet sich für die Grossviehhaltung und natürlich wurde dort damit begonnen; ob langfristig erfolgreich, bleibt noch abzuwarten.

Ueber der Waldgrenze steht die Bodenbedeckung in Abhängigkeit der Dicke und der Andauer der Winterschneedecke. Windexponierte Hänge und Grate sind dauernd frei von Schnee. Pflanzen wachsen hier nicht; es ist das Gebiet der Strukturböden. Normal exponierte Hänge sind mit Latschendickicht (Pinus pumila) überzogen und Mulden zeigen oft Bültenfelder und perennierende Schneeflecken. Die rezente Schneegrenze überragt aber selbst der $3776 \mathrm{~m}$ hohe Fuji San nicht. Der Vertikalabstand von der Wald- zur Schneegrenze beträgt $1300-2000 \mathrm{~m}$. Man vergleiche diesen Wert mit anderen ganzjährigen humiden $\mathrm{Ge}-$ bieten der gemässigten Breiten, die einen weniger extremen Jahresgang der Temperatur aufweisen (K. HERMES 1955).

\section{Rahmen für die Landwirtschaft}

Reis ist das seit alters her wichtigste Anbauprodukt Japans. Da Bewässerung ganzjährig fast überall möglich ist, werden die naturgeographischen Grenzen für die Verbreitung des Reisanbaus in erster Linie durch das Relief und die Temperaturverhältnisse gesetzt (Abb.3). An klimatisch bevorzugten Küstenstreifen im Süden des Landes sind zwei Reisernten pro Jahr die Regel. Im übrigen Teil von Kyushu, Shikoku und WestHonshu sind zwar auch zwei Ernten möglich, doch lässt man in der kühlen Jahreszeit nicht Reis, sondern Weizen oder Gemüse wachsen. Im grössten Teil Honshus ist nur eine Ernte pro Jahr zu erreichen. Im winterlich schneearmen Omote Nippon können in diesem Bereich und dort, wo Weizen auf den Reis folgt, die Felder durch flächenhafte Bodenabtragung gefährdet werden. Ausgelöst wird sie durch Shimobashira (JIYU GAKUEN SCIENCE CROUPE 1937, 1940; L. ELLENBERG 1974b).
Shimobashira (Shimo = Reif; bashira = Säule) ent steht, wenn der nicht durch Schnee isolierte, feuchte poröse Boden nachts oberflächlich gefriert. Eispartikelchen bilden sich in der obersten Bodenkrume; kapillar hochgesaugtes Wasser gefriert zu millimeterdünnen aber mehrere Zentimeter langen Eisnadeln, die die oberste Krume anheben. Kammeis (pipkrake) ist zwar auch in Mitteleuropa bekannt; da dort jedoch der Boden im Winter nach Frost nicht regelmässig wieder tagsüber auftaut oder aber schneebedeckt ist, findet das Auffrieren nicht häufig statt. In Omote Nippon kommen bis zu 40 Nächte mit Shimobashira zwischen Oktober und März vor! Der Wind hat dann ein leichtes Spiel, die gelockerte und angehobene Bodenkrume tagsüber von den pflanzenfreien Ackerflächen abzuwehen. Bis zum 2. Weltkrieg war das "mugi fumi" (= Weizentreten) eine wichtige winterliche Tätigkeit für die Bauern: der Boden wurde nach einer Frostnacht wieder festgetreten. Heute setzt man Walzen für diesen Zweck ein.

In Tohoku, Süd- und Zentral-Hokkaido kommt Reis zwar noch vor, wird aber mit jeder Fahrtstunde in nördlicher Richtung unwichtiger. Der Reisanbau ist hier nur einige Zehner von Jahren oder höchstens hundert Jahre alt. Er wurde erst möglich, nachdem schnell reifende Sorten gezüchtet waren, die nicht zuerst in Saatbeeten herangezogen und dann ungepflanzt werden mussten, sondern die direkt ausgesät werden konnten. Da das japanische Alltagsleben ohne Reis kaum denkbar ist, wird es verständlich, dass Hokkaido als Siedlungsraum für Japaner zunächst nicht in Frage kam. Die dorthin zurückgedrängten früheren

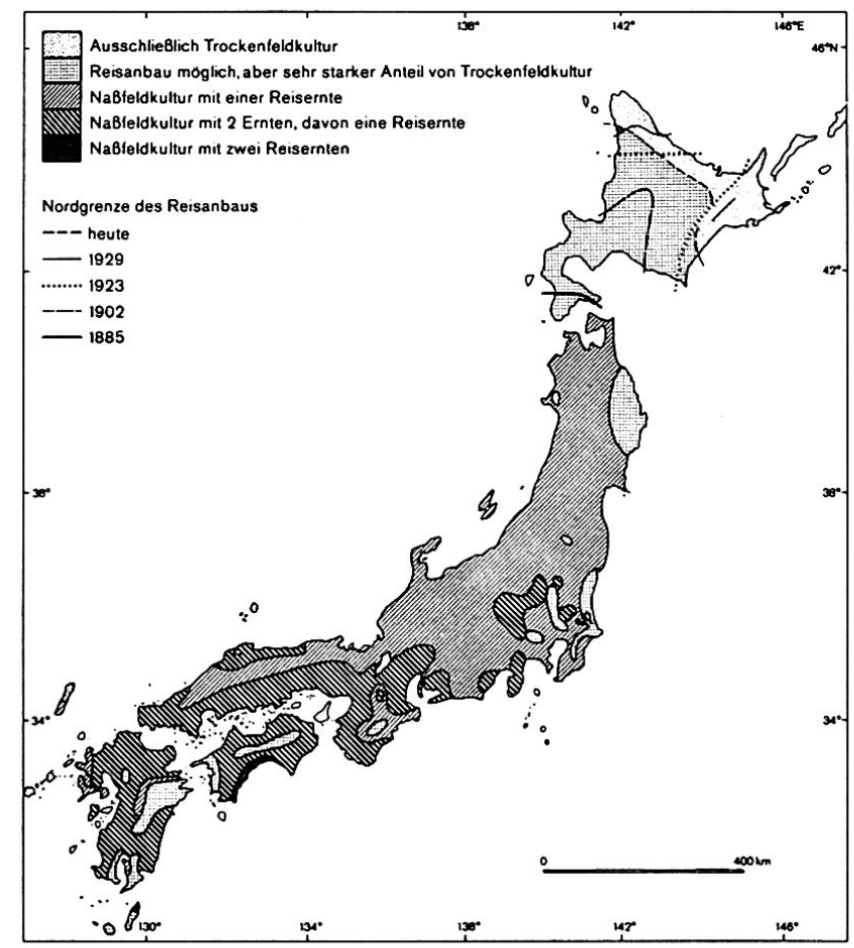

Abb.3: Reisanbau (nach M. SCHWIND 1967) 
Bewohner der Inselkette, die Ainus, wurden zu Ende des letzten und zu Beginn dieses Jahrhunderts systematisch aufgerieben. Zeitgleich und noch jünger als die Ausrottung der Indianer in Nordamerika war diese rücksichtslose Ausweitung des japanischen Agrarraums; allerdings wurde offiziell hierüber nur wenig bekannt.

Dass Hokkaido heute in vieler Hinsicht als Pionierland Japans zu gelten hat, muss meiner Meinung nach zu allererst mit seinem etwas rauheren Klima erklärt werden.

\section{Jahresgang der Sterblichkeit}

In Japan sterben nicht jeden Tag gleichviel Menschen; überblickt man den Jahresgang der Sterblichkeit (K. KATAYAMA and M. MOMIYAMA 1969, 1972) so tritt der Winter und frühe Frühling als Zeit besonders vieler Todesfälle gegenüber dem Sommer hervor. Das Verhältnis ist 130: 70 für die Summe aller Todesfälle und 140 : 60 für die Kindersterblichkeit. Man kennt diesen Jahresgang der Sterblichkeit aus Italien oder Spanien, und er war noch in den Zwanziger Jahren dieses Jahrhunderts in der Schweiz, in England, Deutschland und in den USA festzustellen, wenn auch die Ungleichverteilung niemals so frappierend gross war. Warum ist der Winter in Japan die kritische Zeit für die gebrechlichen Alten und die kleinen Kinder?

Die Antwort wurde in der Einleitung dieses Artikels bereits angedeutet. "Samui desu, nê?" (= kalt ist es, nicht wahr?) drückt mehr aus als ein vorübergehendes Frösteln zu Winterbeginn. Es illustriert eher das Ausgeliefertsein an eine harte Jahreszeit.

Die Häuser sind im sommerheissen Teil Japans den langen feucht-schwülen Wetterlagen gut angepasst. Leichtes Baumaterial wie Holz, Glas, Binsen- und Reisstrohgeflecht, poröse Ziege1, Dachpappe, Blech, Papier und Karton werden verwendet. Ritzen und Fugen bleiben unverdichtet und die eingeschossigen traditionell gebauten Hütten sind meist etwas gestelzt, so dass die Luft unter dem Haus und durch die möbelfreien Räume streichen kann. Von bai-u bis shurin bringt jeder Lufthauch ersehnte Kühlung und ist nötig, damit die Kleider, Wände und Böden nicht stockig werden und verschimmeln. Abgesehen davon, dass dieses Baumaterial leicht Feuer fängt, ist der japanische Baustiel erdbebengerecht.

Der kalten Jahreszeit ist man demgegenüber fast schutzlos preisgegeben. Die kalte Aussenluft sickert ungehindert ins Innere. In Tokyo sind im Januar $+2^{\circ} \mathrm{C}$ am Morgen in den Zimmern keine Seltenheit! Kleine Oelöfelchen, die nachts aus Angst vor Erdbebenerschütterungen abgeschaltet werden, vermögen wenig dagegen zu schützen. Heizbare Schlafdecken sind (noch) nicht weit verbreitet. Der Kotatsu (eine mit einer Tischplatte überdachte Vertiefung im Boden, die mit einer Wärmelampe geheizt wird, in die man die Beine hineingleiten lässt und wo die Wärme mit schweren Decken zwischen Platte und Tatami gehalten wird) ist zwar angenehm, hat aber einen unguten Lagerfeuer-Effekt, denn der Oberkörper bleibt ausserhalb der Wärme.

Ein deutliches Wintermaximum der Sterblichkeit gilt allerdings nicht für den äussersten Süden und Norden des Landes. Dort ist das Verhältnis zwischen Winter- und Sommersterblichkeit höchstens 110 : 90. Diese an prosperierende westliche Industrieländer erinnernden Verhältnisse sind nicht überraschend: in Süd-Kyushu und Hokkaido ist der Winter keine Jahreszeit verminderter Lebensqualität. Der Süden bleibt ganzjährig mild und der Norden wird derart kalt, dass ausreichende Heizung (meist offener Kamin in der Mitte des Wohnraumes) lebensnotwendig ist. Die geheizten Räume machen den Winter in Hokkaido zu einer Jahreszeit, die weit angenehmer ist als beispielsweise in Tokyo.

In Korea schützt man sich seit Jahrhunderten durch eine Fussbodenheizung (Ondol). In der vertieft angelegten Küche bleibt der Herd im Winterhalbjahr dauernd an; der abziehende Rauch wird unter den Wohn- und Schlafkanmern durchgeleitet. Das Ondo1-System hat übrigens bewirkt, dass Korea kaum noch Hochwälder besitzt, denn viel Holz und Holzkohle werden für die lange kalte Jahreszeit benötigt. Wäre eine Uebertragung dieser Fussbodenheizung vom tektonisch stabilen Korea auf das erdbebengefährdete Japan möglich, so wäre der Jahresgang der Sterblichkeit ohne eine Winterspitze.

Nachdenklich mag stimmen, dass der Industriegigant Japan es bis heute noch nicht erreicht hat (noch nicht für nötig erachtete?), dass seine Bürger im Winter wirksam vor Kälte geschützt sind.

\section{Schluss}

Die Prägung Japans durch das Klima ist viel stärker, als man es bei einem so stark vom Menschen in Besitz genommenen Land erwarten würde. Vorangehend wurde angesprochen, wie sich das Klima auf die Formung des Reliefs auswirkt, warum die Japan Sea Seite Honshus noch heute weltabgeschieden wirkt, welche Waldtypen wo entstanden sind, warum rezente Periglazialformen in der obersten Waldstufe nicht die Ausnahme, sondern die Regel darstellen, welcher Rahmen für die Landwirtschaft besteht, warum Hokkaido erst spät japanisiert wurde und warum im Winter in Honshumehr Menschen sterben als im Sommer. Die Beispiele für die Auswirkungen des Klimas lassen sich beliebig vermehren. Das könnte zu einer Ueberbewertung der Prägung durch das Klima verleiten und zu einem einseitigen geographischen Determinismus.

Deshalb sei abschliessend darauf hingewiesen, dass viele Akzente Japans auch ganz anders gedeutet werden sollten. Die Prägung Japans durch die Geologie, durch Shintoismus und Buddhismus, durch die Ferne zu anderen Industriezentren, durch das Desaster des verlorenen Weltkrieges und andere Aspekte sind ähnlich berechtigt und reizvol1. 
ATLAS OF JAPAN, Physical, Economic and Social, Tokyo 1974

ELLENBERG, L.: The periglacial stage in Europe (especially the Alps) and Japan - a comparison, Geogr. Rep. of Tokyo Metropolitan University 9, p. 53-65, 1974a

"Shimobashira - Kammeis in Japan, Geogr. Helv. 29, p. 1-5, 1974b

" Zur Solifluktionsgrenze in den Gebirgen Japans, Erdkunde 31/1, p. 16-24, 1977

HERMES, K.: Die Lage der oberen Waldgrenze in den Gegenden der Erde und ihr Abstand zur Schneegrenze, Kölner Goegr. Arb. 5, 1955

JIYU GAKUEN SCIENCE GROUPE: Untersuchungen über Shimobashira (japanisch), Tokyo, 1. Teil
1937, 2. Teil 1940

KATAYAMA, K. and MOMIYAMA, M.: A biometeorological study in seasonal variation of stroke mortality, Journal Met. Soc. Japan 47, p. 360-372, 1969

" The seasonal variation of stroke mortality and its temperature in Japan, Papers in Met. and Geophysics 23/4, p. 329-345, Tokyo 1972

NUMATA, M.: The flora and vegetation of Japan, Tokyo, Amsterdam, London, New York 1974

SCHWIND, M.: Das Japanische Inselreich, Band 1, Die Naturlandschaft, Berlin 1967

WALTER, H. und LIETH, H.: Klimadiagramm - We1tatlas, Jena, 1. Lieferung 1960

\section{Literaturbesprechungen}

FLUECHTER, Winfried: Stadtplanung in Japan. Problemhintergrund, gegenwärtiger Stand, kritische Bewertung. Mitteilungen des Instituts für Asienkunde, Nr. 97, 125 S., 6 Abb., 7 Tab., 9 Bilder, Selbstverlag des Instituts für Asienkunde, Hamburg, 1978. DM 15,-

Im dichtbesiedelten japanischen Inselreich (113 Mio. Einw./370 $000 \mathrm{~km} 2$ ) sind raumordnende Massnahmen eine conditio sine qua non, zumal sich $57 \%$ der Bevölkerung auf nur 2,2: der Gesamtfläche konzentrieren (1975). Aber erst mit der Sensibilisierung des Umweltbewusstseins seit Ende der 60er Jahr hat ein Demokratisierungsprozess eingesetzt, der nun allmählich das Interesse an öffentlichen Angelegenheiten zu wecken beginnt. Mit dem Planungsgesetz von 1968 (praktiziert seit 1970) sind gewisse planerische Werkzeuge geschaffen worden.

Grösstes Hindernis für eine fortschrittliche Stadtplanung bedeutet heute das Fehlen einer wirksamen Regionalplanung. Nach dem Scheitern der Tanaka-Konzeption ('Umgestaltung des japanischen Archipels", 1972) hat der anhaltende Ballungsprozess die Probleme in den verstädterten Gebieten weiter verschärft. Stadtplanung ist darum kaum mehr als Anpassungsplanung. Sie hat bisher meist nur Tatbestände gutgeheissen. Pla- nerische Tätigkeit vermochte nicht Konzepte zu realisieren - sie konnte nur Vorgänge illustrieren.

Der Autor stellt vor allem geographische, d.h. raumwirksame Teilbereiche von Verstädterung und Stadtplanung in den Mittelpunkt. Probleme wie Flächennutzung, Flächensicherung, Bebauungsbegrenzung und Planungskoordination werden behandelt, Grundstückstruktur und Bauauflagen, Flächenbenutzungsplanung, städtische Infrastruktur Zentrumsbildung oder Koordination von Stadt- und Regionalplanung dargestellt.

Die Ineffizienz der Stadtplanung wird in 7 Punkten zusammengefasst:

- Priorität des Wirtschaftswachstums während langer Zeit

- Grosse, ungebremste Bevölkerungsballungen

- Mangelnde Durchsetzungskraft der Planungsorgane

- Unzureichend detaillierte Planungsziele

- Teils zwielichtiger Einfluss grosser Kapitalgesellschaften

- Misstrauen privater Grundeigentümer

- Fehlen einer wirksamen Regionalplanung.

Das Büchlein ist eine sehr kritische - aber durchwegs positive - Stellungnahme zu einer fast unlösbaren Notsituation.

GH 2/79

Oskar Bär, Zürich 